\title{
Discussion on Landscape and Greening Technology of Highway Structural Tunnel Entrance
}

\author{
Liu ming ${ }^{1}$, Liu Zhiqiang ${ }^{2,3}$, Qiang Rongrong ${ }^{2,3}$ \\ ${ }^{1}$ Hangzhou Qianhuang Expressway Co., Ltd, Zhejiang Province Hangzhou \\ ${ }^{2}$ Research Institute of Highway Ministry of Transport \\ ${ }^{3}$ Key Laboratory of Transportation Industry for Highway Traffic Industry Environmental Protection Technology
}

\begin{abstract}
Highway tunnel entrance is not only the exposed part of mountain in the tunnel engineering, but also an integral part of the highway breaking through the mountain. On the premise of ensuring the safety of tunnel entrance, it is important to realize the unity of safety and practical "stability" and landscape greening "beauty" of the expressway tunnel through reasonable aesthetic landscape greening design. In combination with the topographic and geomorphological conditions as well as the regional and humanistic conditions of the highway tunnel portal area, this paper introduces the cultural landscape and plant greening engineering of highway tunnel entrance by reference to the successful experience of tunnel entrance landscape greening, and puts forward the requirements for landscape and greening technology of highway tunnel entrance in accordance with the provisions of relevant regulations.
\end{abstract}

\section{Background}

Tunnel entrance is the exposed part of mountain in the tunnel engineering. The quality of landscape design directly affects the quality of the whole tunnel and even the whole highway. With the development of economy, the tunnel not only has the single function of traffic, but also performs the publicity function of surrounding cities and scenic spots. These new functions improve the status of tunnel, and the landscape greening design plays an important role in enhancing the regional value. In combination with the human elements, natural elements and social elements of highway tunnel area, this paper puts forward the application principle ${ }^{\mathrm{a}}$, construction process and key points for landscape decoration and greening restoration technology of tunnel entrance area, and provides reference for landscape greening design, construction and research of highway tunnel entrance.

\section{Environmental analysis of tunnel entrance area}

According to the environmental division of tunnel area, the landscape greening of tunnel entrance is divided into two parts: landscape engineering and greening engineering. Based on the regional and humanistic characteristics as well as the landscape segment of the tunnel surrounding area, the special landscape scheme which highlights the cultural theme is customized for the landscape engineering. Eco-friendly materials or bionic materials should be selected as far as possible for decoration and beautification, deliberate modeling of end walls should be avoided, and masonry pollution should be reduced. The greening engineering is focused on restoration of the damaged mountain vegetation. The appropriate greening restoration scheme should be developed according to the topography and soil quality of the site. Local native species should be planted as far as possible, so as to be harmonious with the surrounding original vegetation and to form scenery. The environment of entrance area can be roughly divided into portal morphologic area, entrance side slope and face upward slope area, plane area before entrance, etc.

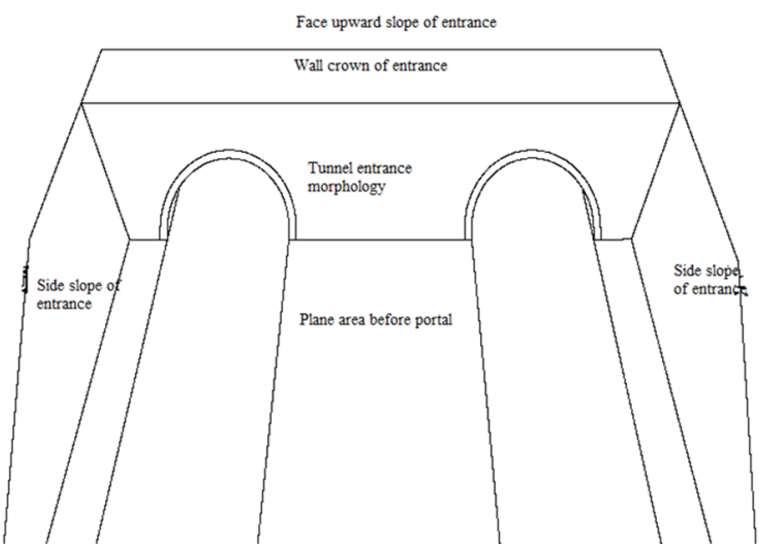

Fig. 1. Environment of tunnel entrance area 


\section{Principles of tunnel entrance landscape design}

\subsection{Safety principle}

In view of the special service groups of highway tunnel, the tunnel entrance landscape design should be carried out based on the safety of motor vehicles, drivers and passengers. The "black-white space" effect when entering and exiting the tunnel entrance should be weakened, and the temporary visual impact caused by the shading transition process should be avoided.

\subsection{Ecological principle}

In the design of tunnel entrance landscape greening, the ecological environment protection and restoration should be priority concerns. Therefore, the ultimate goal of landscape greening is to improve and repair the ecological damage caused by mountain excavation during tunnel construction, combine it with the original mountain, reproduce the natural landform of the mountain, and make landscape greening and nature coexist harmoniously.

\subsection{Regional principle}

Highway tunnel is characterized by spot-like landscape, bearing the history and humanities, natural features, folk tradition and fold customs, cultural economy, etc. With a variety of landscape techniques, the local human elements, natural elements, social elements and other landscape morphologies are integrated into the tunnel entrance arch, end wall, face upward slope and even lighting, fully reflecting the local regional characteristics.

\subsection{Principle of energy efficiency}

In highway tunnels, the energy consumption of lighting brings a great expense at the later operation. Under the premise of meeting the specification requirements, the purpose of cost saving and energy efficiency for the later operation can be achieved by selecting the reasonable landscape design scheme, adopting new technology, new materials and new process, and reducing the ventilation and lighting energy consumption.

\section{Promotion measures of tunnel entrance landscape}

The design of tunnel entrance landscape should be combined with the modeling of the portal, the topography around the portal, as well as the humanistic characteristics and natural environment of the tunnel area.

\subsection{Decoration technology}

The tunnel entrance with portal retaining wall adopts the simple and abstract landscape decoration technique, takes the portal end wall as the carrier, uses the concise patterns and symbols as the expression technique, utilizes the stone-like spray painting process, and reflects the local folk customs and humanity history. The "stone-like spray painting" process is mainly adopted for decoration of the portal wall, the color is generally dark, and the modeling is simple and abstract.

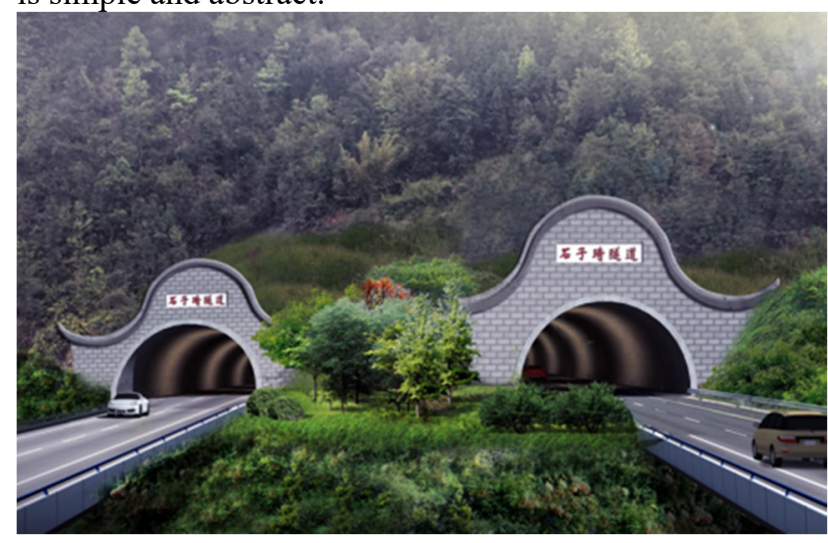

Fig. 2. Ishizaki Tunne

\subsection{Landscaping technology}

For the tunnel entrance without portal retaining wall, on the premise of not affecting the main structure of tunnel construction, the landscape techniques such as "cultural landscape wall" and "landscape stone nameplate" are used to improve the visual landscape of the retaining wall area between two tunnel portals as well as the intermediate belt area of the front separated subgrade, which highlight the tunnel "characteristics" and enhances the recognition of the tunnel. The tunnel portal cultural landscape wall adopts concise modeling and appropriate body shape. For the tunnel entrance without tunnel nameplate, the nameplate landscaping should be arranged at the intermediate belt area of the front separated subgrade.

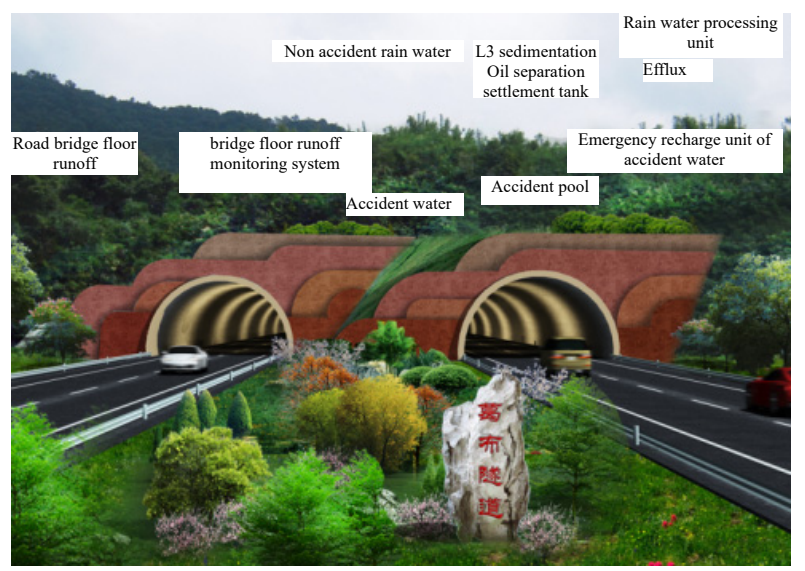

Fig. 3. Gebu Tunnel

\subsection{Landscape application of sunshade at entrance}

Sunshade is arranged at the tunnel entrance for shading and noise reduction, enhancing the visual effect of the entrance landscape. For the sunshade landscape at the tunnel entrance, the sunshade should be reasonably arranged in the principles of safety, energy saving and 
environmental protection for highway tunnel, the structure type of the sunshade should be selected according to the local conditions, and the scale of the sunshade should be controlled within certain limits, so as to improve the driving environment of the tunnel, enhance the driving safety and reduce the operation and maintenance cost. The sunshade landscape should be coordinated with the decoration of portal wall, the length should conform to the functional requirements, landscape requirements or vehicle speed, and the structure type should be concise and beautiful, with clear force-bearing and force-transferring paths.

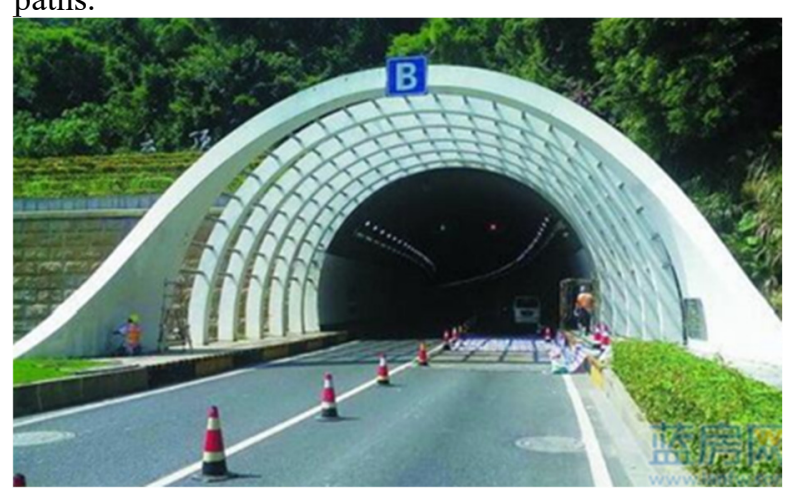

Fig. 4. Tunnel sunshade

\subsection{Application of landscape lighting in tunnel}

Landscape lighting is arranged in the super long tunnels, which can relieve the feeling of driving fatigue and irritability, improve the safety and driving comfort, and create the natural landscape effect in the tunnel. Landscape lighting in the tunnel should conform to the relevant specifications and requirements for tunnel lighting, follow the design principles of advancement, artistic effect, landscape characteristics, diversified expression, orderly environment, centralized control, safety facilities and environmental protection, and use the special intelligent controllers. The power supply system of landscape lighting should be connected to the original power supply system of the tunnel, and the clean energy technology such as solar energy should be adopted as far as possible in order to reduce the power consumption. The energy-saving lamps with long life and aging resistance should be used, the reasonable position of lamps and light source angle should be determined, so as to ensure the lighting effect and avoid glare. The landscape lighting design should be carried out for super long tunnels, which can create the rich visual scene effect, adjust the driving fatigue, irritability, depression and other discomfort feelings, and improve the driving safety and comfort.

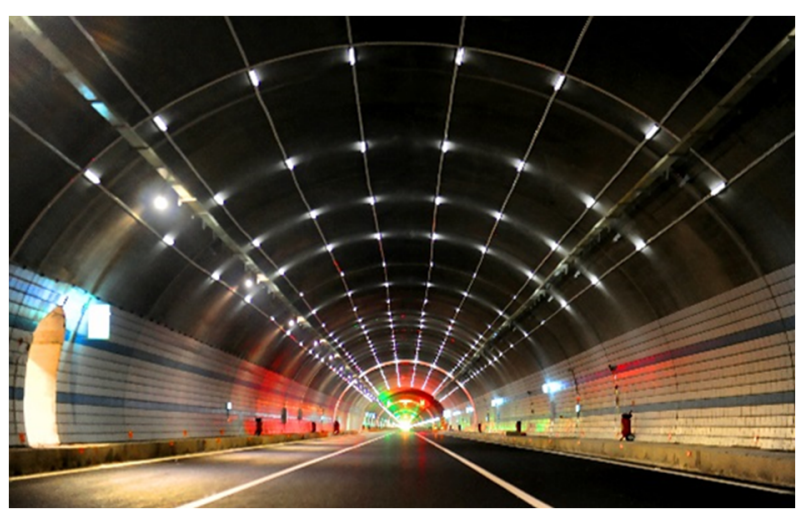

Fig. 5. Landscape lighting in tunnel

\section{Greening technology of tunnel entrance}

For the areas with greening requirements during the tunnel entrance excavation, different ecological restoration technologies should be adopted according to different site conditions, and complex plant communities with diversified structure should be formed as far as possible, with the short-, medium- and long-term effects of side slope greening taken into account.

\subsection{Greening technology of portal facade}

After the completion of tunnel entrance construction, the protective retaining wall with large exposed surface has an impact on the visual landscape. For this area, under the premise of not affecting the portal structure, the hanging net should be used for the exposed protective retaining wall, and the vine plants should be planted on it.

\subsection{Ecological restoration technology of entrance side slope and face upward slope}

After the completion of tunnel portal construction, the vegetation of the surrounding area may be damaged, resulting in the exposed part of mountain in the area. According to different degrees of excavation and different soil conditions, different ecological restoration technologies should be adopted, and complex plant communities with diversified structure should be formed as far as possible, with the short-, medium- and long-term effects of greening taken into account.

5.2.1 For the tunnel entrance slope with good soil quality, slow slope and exposed soil surface, the ecological restoration technology of grass and shrub spot sowing and broadcast sowing is adopted for greening.

\subsubsection{For the tunnel entrance with unstable soil quality, temporary engineering protection or strong weathering unprotected rock slope is designed, and the ecological restoration technology of base material soil spraying is adopted for greening.}


5.2.3For the tunnel entrance slope with slope engineering protection, the ecological restoration technology of planting bag is adopted for greening.

\subsection{Green planting technology of entrance wall crown}

For the tunnel entrance with portal retaining wall, after the completion of construction, there is a certain greening blank area between the portal retaining wall and the mountain. For this area, on the premise of not affecting the tunnel structure, small trees, shrubs, vines, herbs and other different levels of vegetation are used for greening according to the site soil and backfill quality.

\subsection{Greening technology of plane area before portal}

The greening area before the tunnel portal consists of two parts: the separated subgrade intermediate belt and the subgrade side. Firstly, the greening layout should be carried out in a targeted manner, with different plants used as the base tone, so as to match the prominent features, enhance the regional characteristics and identification, and form an unique plant greening theme. Secondly, in consideration of the light reduction effect of vegetation, the brightness of the tunnel entrance area facade can be reduced by virtue of soft reflection and human visual comfort of the vegetation. The shadows cast by tall trees are utilized to weaken the sunlight towards the road surface, thus reducing the brightness of the plane area before tunnel entrance.

\section{Conclusion}

The landscape greening engineering of highway structural tunnel entrance shows that the safety of tunnel portal is not only the single function. On the premise of ensuring the safety of tunnel portal, a beautiful landscape can be created through reasonable aesthetic landscape design, which provides the visual and cultural presentation of the tunnel portal - an important landscape node of the highway. To adhere to the concept of green traffic development and put the technical measures of green highway into effect, the goal of tunnel entrance greening is achieved through the technical approach and implementation method of tunnel entrance landscape and greening technology. The core of tunnel entrance landscape and greening technology is to integrate the tunnel entrance landscape into the natural environment by combining the restoration and renovation technology of tunnel entrance landscape with the ecological restoration technology of facade plane and by organizing and implementing the specific construction scheme after determining the greening elements of tunnel entrance landscape.

\section{Contact information}

Liu Zhiqiang 13811592577, Environmental Center of Research Institute of Highway Ministry of Transport

\section{Acknowledgement}

${ }^{\text {a}}$ Scientific research projects of Zhejiang Provincial Department of Communications: Technologies of green environmental protection and operation safety of Huqu Expressway (2020-GCKY-01)

\section{References}

1. LIU Fei. Road tunnel portal selection based on environmental protection. Chongqing. (Chongqing Jiaotong University, 2010).

2. JIA Lingli, ZHAO Dongping. A study on the trend of development and present state of tunnel entrance landscaping. China Civil Engineering Journal, 41,1(2008).

3. YE Fei, XU Changxin, WEI Yanchun, et al. Aesthetic analysis of mountain highway tunnel entrance. Tunnel Construction, 2019, 39,4(2019).

4. LI Guofeng, MA Guomin, LI Wenhui, et al. Study ondynamic landscape design issue of highway tunnel portal. Highway, 63,12(2018).

5. GUAN Xiang-qun. The Research of a Practical Method of Aesthetics Design in the Tunnel Entrance. (Southwest Jiaotong University, 2004). 